\title{
9. COMMISSION DES INSTRUMENTS ASTRONOMIQUES
}

\author{
Compte rendu de la séance. 3I août 1955
}

Président intérimaire: Prof. Ch. Fehrenbach.

SECRÉTAIRE: Dr W. L. Wilcock.

Après avoir transmis les regrets du Président de la Commission 9, Monsieur A. Couder, empêché par des raisons de santé, le Président de la séance rappelle la suggestion qui a été faite par divers membres de diviser la Commission en deux nouvelles Commissions. L'une des Commissions s'occuperait des instruments d'optique, l'autre, des instruments électroniques. Il signale que dans l'opinion de M. Couder, une telle subdivision est artificielle et contre-indiquée. Comme d'autre part le mandat de M. Couder arrive à expiration, après six années de présidence, il suggère qu'on élise, comme nouveau président, un astronome spécialisé en électronique. Le R.P. Treanor est d'accord avec M. Couder pour considérer la subdivision comme contre-indiquée, car tous les membres s'intéressent à des appareils collectant et détectant les radiations. Il n'y a pas d'autre discussion sur ce sujet. Le Président propose alors le nom du Dr Kron pour succéder au Dr Couder, comme Président de la Commission. Cette proposition est acceptée à l'unanimité.

Le Président remarque que le travail de la Commission est simplifié parce que deux Symposia sur les instruments ont lieu en 1955 :

I. Un Symposium sur les Convertisseurs d'images électroniques a lieu pendant le Congrès de Dublin.

2. Un certain nombre d'instruments d'optique nouveaux ont été décrits dans un Symposium sur les Instruments d'Optique Astronomique qui s'est tenu en avril à Manchester, et les travaux de ce Congrès seront publiés et distribués dans un bref délai.

Les rapports suivants ont été faits verbalement:

La coupole du télescope de $193 \mathrm{~cm}$. de l'Observatoire de Haute-Provence est presque achevée et l'instrument et ses accessoires seront en service en 1956.

Le télescope de $120 \mathrm{~cm}$. de l'Observatoire de Crimée est en service.

Un télescope de Schmidt, de $72 / 90 \mathrm{~cm}$. est en construction pour l'Observatoire de Rome. Les pièces d'optique sont terminées et la partie mécanique sera achevée en 1956. L'instrument sera placé sur le Gran Sasso, à une altitude de 2 I $22 \mathrm{~m}$.

Un nouvel observatoire est en construction à Caracas (Observatoire Astronomique, Séismologique et Géomagnétique, Cagigal Caracas, Directeur: Dr Eduardo Röhl, latitude = $10^{\circ} 30^{\prime} 24^{\prime \prime} \mathrm{N}$., longitude $=4^{\mathrm{h}} 27^{\mathrm{m}} \mathrm{W}$.). L'instrument principal sera un réfracteur de $65 \mathrm{~cm}$. de Zeiss, ayant une longeur focale de $1050 \mathrm{~cm}$., un réflecteur de $100 \mathrm{~cm}$. de Zeiss avec des foyers Cassegrain et Coudé, un télescope de Schmidt de $65 / 94 \mathrm{~cm}$. (Askania), une lunette méridienne de $190 \mathrm{~cm}$. de distance focale (Askania), une lunette zénithale photographique (Askania), un instrument des passages (Askania), trois horloges à quartz et deux horloges à pendule. On attire spécialement l'attention sur le fait que cet observatoire est très près de l'Équateur.

Le télescope de Schmidt, de l'Observatoire royal de Belgique à Uccle, de 83/120 cm., doit être mis en service en 1956. Le télescope de 74 inches, de l'Observatoire d'Helwan (Égypte), qui sera équipé avec des spectrographes Cassegrain et Coudé, sera mis en service dans deux ans.

Les instruments en construction ou récemment achevés, à l'Observatoire Ondrejov, de l'Institut Astronomique de l'Académie des Sciences Tchécoslovaque, comprennent une tour solaire avec spectrographe, filtre polarisant d'un type différent de celui de Lyot, un télescope radio-électrique de $7^{\mathrm{m}} \cdot{ }_{5}$ de diamètre travaillant sur une longueur d'onde de $5^{8} \mathrm{~cm}$., deux photomètres pour l'observation du ciel nocturne et un télescope de Schmidt de $60 / 40 \mathrm{~cm}$. 